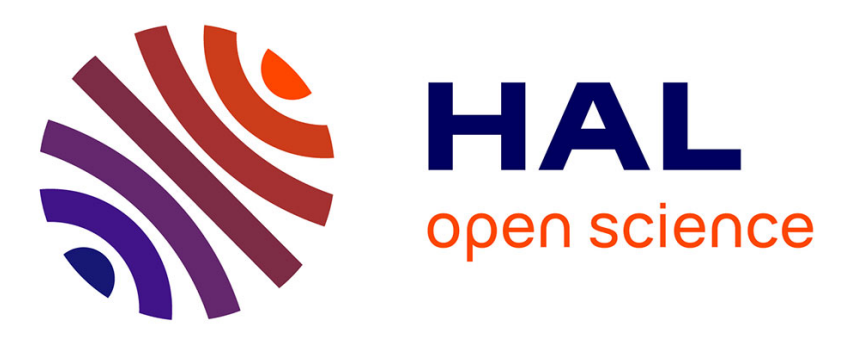

\title{
Morphological Semigroups and Scale-Spaces on Ultrametric Spaces
}

Jesus Angulo, Santiago Velasco-Forero

\section{To cite this version:}

Jesus Angulo, Santiago Velasco-Forero. Morphological Semigroups and Scale-Spaces on Ultrametric Spaces. 13th International Symposium on Mathematical Morphology and Its Applications to Signal and Image Processing, May 2017, Fontainebleau, France. pp.28-39, 10.1007/978-3-319-57240-6_3 . hal-01536368

\section{HAL Id: hal-01536368 \\ https://hal-mines-paristech.archives-ouvertes.fr/hal-01536368}

Submitted on 11 Jun 2017

HAL is a multi-disciplinary open access archive for the deposit and dissemination of scientific research documents, whether they are published or not. The documents may come from teaching and research institutions in France or abroad, or from public or private research centers.
L'archive ouverte pluridisciplinaire HAL, est destinée au dépôt et à la diffusion de documents scientifiques de niveau recherche, publiés ou non, émanant des établissements d'enseignement et de recherche français ou étrangers, des laboratoires publics ou privés. 


\title{
Morphological semigroups and scale-spaces on ultrametric spaces
}

\author{
Jesús Angulo Santiago Velasco-Forero \\ MINES ParisTech, PSL-Research University, \\ CMM-Centre de Morphologie Mathématique, France \\ jesus. angulo@mines-paristech.fr
}

\begin{abstract}
Ultrametric spaces are the natural mathematical structure to deal with data embedded into a hierarchical representation. This kind of representations is ubiquitous in morphological image processing, from pyramids of nested partitions to more abstract dendograms from minimum spanning trees. This paper is a formal study of morphological operators for functions defined on ultrametric spaces. First, the notion of ultrametric structuring function is introduced. Then, using as basic ingredient the convolution in ( $\max , \min )$-algebra, the multi-scale ultrametric dilation and erosion are defined and their semigroup properties are stated. It is proved in particular that they are idempotent operators and consequently they are algebraically ultrametric closing and opening too. Some preliminary examples illustrate the behavior and practical interest of ultrametric dilations/erosions.
\end{abstract}

Keywords: ultrametric space; ultrametric semigroup; idempotent operator; (max,min)-convolution

\section{Introduction}

Morphological operators are classically defined for real-valued functions supported on Euclidean or Riemannian spaces [1] and are used for nonlinear image processing. More recently, morphological semigroups for functions on length spaces have been studied [3], whose basic ingredients are the convolution in the (max, +)-algebra (or supremal convolution), the metric distance and a convex shape function. More precisely, given a length space $(X, d)$, a bounded function $f: X \mapsto \mathbb{R}$ and an increasing convex one-dimensional (shape) function $L: \mathbb{R}_{+} \rightarrow \mathbb{R}_{+}$such that $L(0)=0$, the multiscale dilation $D_{L ; t} f$ and erosion $E_{L ; t} f$ operators of $f$ on $(X, d)$ according to $L$ at scale $t>0$ are defined as

$$
\begin{array}{ll}
D_{L ; t} f(x)=\sup _{y \in X}\left\{f(y)-t L\left(\frac{d(x, y)}{t}\right)\right\}, & \forall x \in X, \\
E_{L ; t} f(x)=\inf _{y \in X}\left\{f(y)+t L\left(\frac{d(x, y)}{t}\right)\right\}, & \forall x \in X .
\end{array}
$$

A typical example of a shape function is $L(q)=q^{P} / P, P>1$, such that the canonical shape function corresponds to the case $P=2: L(d(x, y) / t)=$ 
$d(x, y)^{2} / 2 t^{2}$. The corresponding semigroups are just: $D_{L ; t} D_{L ; s} f=D_{L ; t+s} f$ and $E_{L ; t} E_{L ; s} f=E_{L ; t+s} f$. These semigroups lead to powerful scale-space properties for multiscale filtering, regularization and feature extraction. The goal of this paper is to consider a similar generalization of morphological semigroups to the case of functions on ultrametric spaces.

An ultrametric space is a special kind of metric space in which the triangle inequality is replaced with the stronger condition $d(x, z) \leq \max \{d(x, y), d(y, z)\}$. Several typical properties on the corresponding ultrametric balls are directly derived from this ultrametric triangle inequality, which lead to nested partitions of the space. Related to the latter property, every finite ultrametric space is known to admit a natural hierarchical description called a dendogram, also known as downward tree. Dendrograms represent a tree structure of the data, where the data points are the leaves of the tree and the vertical axis reveals the ordering of the objects into nested clusters of increasing ordering. Datasets endowed with a hierarchical classification structure are nowadays used in many challenging problems; like the case of very high dimensional spaces where the data structure is generally given by cluster-like organization [12]. In the case of morphological image processing, hierarchical representations are ubiquitous $[15,6,10]$.

Processing a function whose domain is such hierarchical representation requires the formulation of filters and operators on ultrametric spaces. The counterpart of Heat kernel and Heat semigroups on ultrametric spaces has been widely studied in recent work [4] (for discrete ultrametric spaces) and [5] (for complete ultrametric spaces). Indeed using the theory of $[4,5]$, diffusion-based signal/image processing techniques can be applied to filter out functions on a hierarchy. A similar counterpart of morphological signal/image processing for such representations is developed in this paper.

Our starting point is the notion convolution of two functions in the (max, min)algebra. Using this operator, we have recently shown that morphological operators on Euclidean spaces are natural formulated in (max, min)-algebra [2]. We introduce (max, min)-convolution based morphological operators on ultrametric spaces, where the structuring functions are scaled versions of the ultrametric distance (raised to a power $p \geq 1$ ). We study the corresponding semigroups properties and illustrate their interest in filtering and feature extraction.

In the state-of-the-art on mathematical morphology, there are several research lines related to our work. On the one hand, the theory of adjunctions on the lattice of dendograms [8]. We remark that in our framework, the operators will be defined on the lattice of functions on the ultrametric space and not in the lattice of dendograms itself. On the other hand, the various adjunctions on edge or node weighted graphs and their interpretations in terms of flooding $[7,9,13]$ and their application to construct segmentation algorithms from invariants of processed minimum spanning trees with associated morphological operators $[10,11]$. 


\section{Ultrametric spaces}

Let $(X, d)$ be a metric space. The metric $d$ is called an ultrametric if it satisfies the ultrametric inequality, i.e., $d(x, y) \leq \max \{d(x, z), d(z, y)\}$, that is obviously stronger than the usual triangle inequality. In this case $(X, d)$ is called an ultrametric space. An ultrametric space $(X, d)$ is called discrete if the set $X$ is: i) countable, ii) all balls $B_{r}(x)$ are finite, and iii) the distance function $d$ takes only integer numbers.

Properties of ultrametric balls. Let us consider some well known properties of ultrametric spaces that directly derive from the ultrametric triangle inequality, proofs can be find in any basic reference on the field. The intuition behind such seemingly strange effects is that, due to the strong triangle inequality, distances in ultrametrics do not add up.

(1) The strict ball $B_{<r}(x)$ as well as the non-strict ball $B_{\leq r}(x)$ are both open as well as closed sets for the topology defined by the metric.

(2) Every point inside a ball is its center, i.e., if $d(x, y)<r$ then $B_{r}(x)=B_{r}(y)$.

(3) Given three points $x, y, z \in X$,

$$
\begin{aligned}
y, z \in B_{r}(x) & \Rightarrow d(y, z)<r, \\
y \in B_{r}(x), \quad z \notin B_{r}(x) & \Rightarrow d(y, z) \geq r .
\end{aligned}
$$

(4) For two intersecting balls, one contains the other, i.e., if $B_{r}(x) \cap B_{s}(y) \neq \emptyset$ then either $B_{r}(x) \subseteq B_{s}(y)$ or $B_{s}(y) \subseteq B_{r}(x)$.

(5) Any two ultrametric balls of the same radius $r$ are either disjoint or identical.

(6) The set of all open balls with radius $r$ and center in a closed ball of radius $r>0$ forms a partition of the latter, and the mutual distance of two distinct open balls is again equal to $r$.

Consequently, the collection of all distinct balls of the same radius $r$ forms a partition $X$; for increasing values of $r$, the balls are also increasing, hence we obtain a family of nested partitions of $X$ which forms a hierarchy.

Examples of ultrametric spaces. The p-adic numbers form a complete ultrametric space. The Cantor set, which is a fractal model, is also an ultrametric space. Besides these examples, we are interested for our applications in the duality between discrete ultrametric spaces and downward (or rooted) trees, which are also known as dendograms.

We can introduce formally a downward tree $\Gamma$ as follows. Let $\Gamma$ be a countable connected graph, where the set of vertices of $\Gamma$ consists of disjoint union of subsets $\left\{\Gamma_{k}\right\}_{k=0}^{\infty}$ with the following properties: i) from each vertex $v \in \Gamma_{k}$ there is exactly one edge to $\Gamma_{k+1}$; ii) for each vertex $v \in \Gamma_{k}$ the number of edges connecting $v$ to $\Gamma_{k-1}$ is finite and positive, provided $k \geq 1$; iii) if $|k-l| \neq 1$ then there is no edges between vertices of $\Gamma_{k}$ and $\Gamma_{l}$. Let $d_{\Gamma}(v, w)$ denote the graph distance between the vertices $v$ and $w$ of graph $\Gamma$, i.e., the smallest number of edges in a path connecting the two vertices. For two vertices, $x, y \in \Gamma_{0}$, their nearest common ascestor is the vertex $a \in \Gamma_{k}$. Note that $a$ is connected to $x$ and $y$ by downward paths of $k$ edges. Then $\left(\Gamma_{0}, d\right)$ is a discrete ultrametric space. 
Dually, any discrete ultrametric space $(X, d)$ admits a representation as the bottom (i.e., set of leaves) of a downward tree $\Gamma$. Define the vertices of $\Gamma$ to be all distinct balls $\left\{B_{k}(x)\right\}$ where $x \in X$ and $k \in \mathbb{Z}_{+}$. Two balls $B_{k}(x)$ and $B_{l}(y)$ are connected by an edge in $\Gamma$ if $|k-l| \neq 1$ and one of them is subset of the other. That is $\Gamma_{0}$ coincides with the set $X, \Gamma_{1}$ consists of balls of radii 1 , etc. Clearly, edges exist only between the vertices of $\Gamma_{k}$ and $\Gamma_{k+1}$. All balls of a given radius $k$ provide a partition of $\Gamma_{0}$, so that $\Gamma_{k}$ consists of the elements of the partition. Each of the balls of radius $k$ is partitioned into finetely many smaller balls of radius $k-1$ and is contained in exactly one ball of radius $k+1$. The ultrametric distance can be also defined as $d(x, y)=\min \left\{k: y \in B_{k}(x)\right\}$.

\section{Dilation and erosion semigroups on ultrametric spaces}

Functions on ultrametric spaces. Given a separable and complete ultrametric space $(X, d)$, let us consider the family of non-negative bounded functions $f$ on $(X, d), f: X \rightarrow[0, M]$. The complement (or negative) function of $f$, denoted $f^{c}$, is obtained by the involution $f^{c}(x)=M-f(x)$. The set of non-negative bounded functions on ultrametric space is a lattice with respect to the pointwise maximum $\vee$ and minimum $\wedge$.

\subsection{Ultrametric structuring functions}

Definition 1. A parametric family $\left\{b_{t}\right\}_{t>0}$ of functions $b_{t}: X \times X \rightarrow(-\infty, M]$ is called by us an ultrametric structuring function in the ultrametric space $(X, d)$ if the following conditions are satisfied for all $x, y \in X$ and for all $t, s>0$ :

(1) Total mass inequality: $\sup _{y \in X} b_{t}(x, y) \leq M$

(2) Completeness (or conservative): $b_{t}(x, x)=M$

(3) Symmetry: $b_{t}(x, y)=b_{t}(y, x)$

(4) A structuring function is monotonically decreasing in the ultrametric distance.

(5) The complement of the structuring function, i.e., $b_{t}^{c}(x, y)=M-b_{t}(x, y)$, is an ultrametric distance in $\left(X, b_{t}^{c}\right)$

(6) Maxmin semigroup property:

$$
b_{\max (t, s)}(x, y)=\sup _{z \in X}\left\{b_{t}(x, z) \wedge b_{s}(z, y)\right\} .
$$

Let us in particular introduce the so-called natural isotropic structuring function $b_{P, t}(x, y)=b_{P, t}(d(x, y)), P>0$, in $(X, d)$ as the following strictly monotonically decreasing function whose "shape" depends on power $P$ :

$$
b_{P, t}(x, y)=M-\left(\frac{d(x, y)}{t}\right)^{P} .
$$

The case $P=1$ is considered the canonical ultrametric structuring function. 
Proposition 1. For any $P>0$, the isotropic P-power function $b_{P, t}(x, y)$ is an ultrametric structuring function.

Proof. The properties 1 (total mass inequality), 2 (completeness) and 3 (symmetry) are obvious from the definition of ultrametric distance. For property 4 , the $\mathrm{P}$-power function is also clearly monotonically increasing in the ultrametric distance and vanishes at 0 .

For property 5 , we need to prove that if $(X, d)$ is an ultrametric space, then $\left(X, b_{t}^{c}\right)$ is an ultrametric space too, with $b_{P, t}^{c}(x, y)=t^{-P} d(x, y)^{P}, t>0, P>0$. Let $x, y, z \in X$ be given, we have $d(x, y) \leq \max \{d(x, z), d(z, y)\}$. Clearly,

$$
t^{-P} d(x, y)^{P} \leq t^{-P} \max \{d(x, z), d(z, y)\}^{P}=\max \left\{t^{-P} d(x, z)^{P}, t^{-P} d(z, y)^{P}\right\}
$$

Thus $t^{-P} d(x, y)^{P}$ is an ultrametric on $X$.

For property 6 on max-semigroup in the ( $\max , \min )$-convolution, we will use well-known results from this convolution [2]. First, let write the function $f$ by its strict lower level sets:

$$
f(x)=\inf \left\{\lambda: x \in Y_{\lambda}^{-}(f)\right\},
$$

where $Y_{\lambda}^{-}(f)=\{x \in X: f(x)<\lambda\}$. In fact, given $t, s>0$ we will prove the dual semigroup property

$$
b_{P, \max (t, s)}^{c}(x, y)=\inf _{z \in X}\left\{b_{P, t}^{c}(x, z) \vee b_{P, s}^{c}(z, y)\right\} .
$$

which is just equivalent to the one we have since:

$$
\sup _{z \in X}\left\{b_{P, t}(x, z) \wedge b_{P, s}(z, y)\right\}=M-\inf _{z \in X}\left\{t^{-P} d(x, z)^{P} \vee s^{-P} d(z, y)^{P}\right\} .
$$

Without the loss of generality, we can fix $P=1$. By using the classical result from level set representations

$$
Y_{\lambda}^{-}\left(\phi_{1} \vee \phi_{2}\right)=\left\{x \in X: \phi_{1}(x)<\lambda \text { and } \phi_{2}(x)<\lambda\right\}=Y_{\lambda}^{-}\left(\phi_{1}\right) \cap Y_{\lambda}^{-}\left(\phi_{2}\right),
$$

the condition, $\forall x, y \in X$,

$$
Y_{\lambda}^{-}\left(t^{-1} d(x, z) \vee s^{-1} d(z, y)\right)=\left\{\exists z \in X:\left[t^{-1} d(x, z) \vee s^{-1} d(z, y)\right]<\lambda\right\},
$$

becomes $\left\{Y_{\lambda}^{-}\left(t^{-1} d(x, z)\right) \cap Y_{\lambda}^{-}\left(s^{-1} d(z, y)\right) \neq \emptyset\right\}$, or equivalently, $\forall x, y \in X$, $\exists z \in X$ such that $B_{\lambda t}(z) \cap B_{\lambda s}(z) \neq \emptyset$. In addition, $B_{\lambda t}(x)=B_{\lambda t}(z), B_{\lambda s}(y)=$ $B_{\lambda s}(z)$. Using the properties of ultrametric balls, the intersection of two balls centered at $z$ means that there is ball which contains the other of radius $\lambda \max (t, s)$ and that $x$ and $y$ belongs to this ball, i.e.,

$$
\{d(x, y)<\lambda \max (t, s)\} \neq \emptyset .
$$

In conclusion,

$$
Y_{\lambda}^{-}\left(t^{-1} d(x, z) \vee s^{-1} d(z, y)\right)=Y_{\lambda}^{-}\left(\max (t, s)^{-1} d(x, y)\right),
$$

and therefore: $\inf _{z \in X}\left\{b_{t}^{c}(x, z) \vee b_{s}^{c}(z, y)\right\}=b_{\max (t, s)}^{c}(x, z)$. 


\subsection{Ultrametric dilation and erosion multiscale operators}

Definition 2. Given an ultrametric structuring function $\left\{b_{t}\right\}_{t>0}$ in $(X, d)$, for any non-negative bounded function $f$ the ultrametric dilation $D_{t} f$ and the ultrametric erosion $E_{t} f$ of $f$ on $(X, d)$ according to $b_{t}$ are defined as

$$
\begin{array}{ll}
D_{t} f(x)=\sup _{y \in X}\left\{f(y) \wedge b_{t}(x, y)\right\}, \quad \forall x \in X, \\
E_{t} f(x)=\inf _{y \in X}\left\{f(y) \vee b_{t}^{c}(x, y)\right\}, \quad \forall x \in X .
\end{array}
$$

We can easily identify that the ultrametric dilation is a kind of convolution in (max, min)-algebra of function $f$ by $b_{t}$.

Proposition 2. Ultrametric dilation $D_{t} f$ and erosion $E_{t} f$ have the following properties.

(1) Commutation with supremum and infimum. Given a set of functions $\left\{f_{i}\right\}, i \in I$ and $\forall x \in X, \forall t>0$, we have

$$
D_{t}\left(\bigvee_{i \in I} f_{i}(x)\right)=\bigvee_{i \in I} D_{t} f_{i}(x) ; \quad E_{t}\left(\bigwedge_{i \in I} f_{i}(x)\right)=\bigwedge_{i \in I} E_{t} f_{i}(x)
$$

(2) Increasingness. If $f(x) \leq g(x), \forall x \in X$, then

$$
D_{t} f(x) \leq D_{t} g(x) ; \text { and } E_{t} f(x) \leq E_{t} g(x), \forall x \in X, \forall t>0 .
$$

(3) Extensivity and anti-extensivity

$$
D_{t} f(x) \geq f(x) ; \text { and } E_{t} f(x) \leq f(x), \forall x \in X, \forall t>0 .
$$

(4) Duality by involution. For any function $f$ and $\forall x \in X$, one has

$$
D_{t} f(x)=\left[E_{t} f^{c}(x)\right]^{c} ; \text { and } E_{t} f(x)=\left[D_{t} f^{c}(x)\right]^{c}, \forall t>0 .
$$

(5) Ordering property. If $0<s<t$ then $\forall x \in X$

$$
\inf _{X} f \leq E_{t} f(x) \leq E_{s} f(x) \leq f(x) \leq D_{s} f(x) \leq D_{t} f(x) \leq \sup _{X} f .
$$

(6) Semigroup. For any function $f$ and $\forall x \in X$, and for all pair of scales $s, t>0$,

$$
\begin{aligned}
D_{t} D_{s} f & =D_{\max (t, s)} f ; \\
E_{t} E_{s} f & =E_{\max (t, s)} f .
\end{aligned}
$$

Proof. For property 1, on the distributivity of the operators, we have for all $x \in X$ and for $t$ :

$$
\begin{aligned}
D_{t}\left(\bigvee_{i \in I} f_{i}(x)\right) & =\sup _{y \in X}\left\{\sup _{i \in I} f_{i}(y) \wedge b_{t}(x, y)\right\} \\
& =\sup _{i \in I} \sup _{y \in X}\left\{f_{i}(y) \wedge b_{t}(x, y)\right\}=\bigvee_{i \in I} D_{t} f_{i}(x) .
\end{aligned}
$$


and similarly for the ultrametric erosion.

The properties 2 and 3 of increasingness and extensivity/anti-extensivity are obvious from the properties of supremum/infimum and the property $b_{t}(x, y) \leq$ $M$, with $b_{t}(x, x)=M$.

For property 4 , on duality by involution, let us prove the first relationship since the other one is obtained by a similar procedure. For all $x \in X$ and for $t$ :

$$
\begin{aligned}
{\left[E_{t} f^{c}(x)\right]^{c} } & =M-\inf _{y \in X}\left\{(M-f(y)) \vee b_{t}^{c}(x, y)\right\} \\
& =M-\inf _{y \in X}\left\{(M-f(y)) \vee\left(M-b_{t}(x, y)\right)\right\} \\
& =-\inf _{y \in X}\left\{-f(y) \vee-b_{t}(x, y)\right\}=\sup _{y \in X}\left\{f(y) \wedge b_{t}(x, y)\right\}=D_{t} f(x) .
\end{aligned}
$$

In order to prove the semigroup property 6 , let us focus on the ultrametric dilation $D_{t}$. For any $x \in X$ and any pair $t, s>0$, one has:

$$
\begin{aligned}
D_{t} D_{s} f(x) & =\sup _{y \in X}\left[D_{s} f(y) \wedge b_{t}(x, y)\right] \\
& =\sup _{y \in X}\left[\sup _{z \in X}\left\{f(z) \wedge b_{s}(y, z)\right\} \wedge b_{t}(x, y)\right] \\
& =\sup _{z \in X}\left[f(z) \wedge \sup _{y \in X}\left\{b_{s}(y, z) \wedge b_{t}(x, y)\right\}\right] .
\end{aligned}
$$

Then using the property (6) of semigroup for ultrametric structuring functions, it is obtained that

$$
D_{t} D_{s} f(x)=\sup _{z \in X}\left\{f(z) \wedge b_{\max (t, s)}(x, z)\right\}=D_{\max (t, s)} f(x) .
$$

The result for the ultrametric erosion is just obtained by duality.

The proof of ordering property 5 for the case $D_{t}(f)(x) \geq D_{s}(f)(x), \forall x \in X$ is based on the fact for $t>s>0$, by the semigroup property on the structuring functions, one has

$$
b_{t}(x, y)=\sup _{z \in X}\left\{b_{t}(x, z) \wedge b_{s}(z, y)\right\} \Rightarrow b_{t}(x, y) \geq b_{s}(x, y),
$$

and therefore

$$
D_{t} f(x)=\sup _{y \in X}\left\{f(y) \wedge b_{t}(x, y)\right\} \geq \sup _{y \in X}\left\{f(y) \wedge b_{s}(x, y)\right\}=D_{s} f(x) .
$$

Considering the classical algebraic definitions of morphological operators [16] for the case of ultrametric semigroups $\left\{D_{t}\right\}_{t \geq 0}$, resp. $\left\{E_{t}\right\}_{t \geq 0}$, they have the properties of increasingness and commutation with supremum, resp. infimum, which involves that

$D_{t}$ is a dilation and $E_{t}$ is an erosion. 
In addition, they are extensive, resp. anti-extensive, operators and, by the supremal semigroups, both are idempotent operators, i.e., $D_{t} D_{t}=D_{t}$ and $E_{t} E_{t}=E_{t}$, which implies that

$D_{t}$ is a closing and $E_{t}$ is an opening.

Finaly, their semigroups are just the so-called granulometric semigroup [16] and therefore

$$
\left\{D_{t}\right\}_{t \geq 0} \text { is an anti-granulometry and }\left\{E_{t}\right\}_{t \geq 0} \text { is a granulometry, }
$$

which involve interesting scale-space properties useful for filtering and decomposition.

At first sight, one can be perplexed by this property: ultrametric dilation (resp. ultrametric erosion) is also a closing (resp. opening), since ultrametric dilation commutes with the supremum and the class of invariants of a closing is stable by infimum. However, as these suprema are taken on ultrametric balls of the various partitions, their class is also stable by infimum. The same result was already obtained by Meyer [8] for set operators on partitions.

Note that we do not use the duality by adjunction to link this pair of dilation/erosion since they are already idempotent operators and do not need to compose them to achieve such goal. Reader interested on adjunction in (max, min)algebra is referred to [2].

\subsection{Discrete ultrametric dilation and erosion semigroups}

Let $(X, d)$ be a discrete ultrametric space. Choose a sequence $\left\{c_{k}\right\}_{k=0}^{\infty}$ of positive reals such that $c_{0}=0$ and $c_{k+1}>c_{k} \geq 0, k=0,1, \cdots$. Then, given $t>0$, ones defines the sequence $\left\{b_{k, t}\right\}_{k=0}^{\infty}$, such that

$$
b_{k, t}=M-t^{-1} c_{k} .
$$

Let us define $\forall k, \forall x \in X$, the ultrametric dilation and erosion of radius $k$ on the associated partition as

$$
\begin{aligned}
\mathrm{Q}_{k}^{\vee} f(x) & =\sup _{y \in B_{k}(x)} f(y), \\
\mathrm{Q}_{k}^{\wedge} f(x) & =\inf _{y \in B_{k}(x)} f(y) .
\end{aligned}
$$

Using now (6) and (7), it is straightforward to see that the ultrametric dilation and ultrametric erosion of $f$ by $b_{k, t}$ can be written as

$$
\begin{aligned}
& D_{t} f(x)=\sup _{0 \leq k \leq \infty}\left\{\mathrm{Q}_{k}^{\vee} f(x) \wedge b_{k, t}\right\}, \\
& E_{t} f(x)=\inf _{0 \leq k \leq \infty}\left\{\mathrm{Q}_{k}^{\wedge} f(x) \vee\left(M-b_{k, t}\right)\right\} .
\end{aligned}
$$

It is obvious using this formulation that do not need to compute explicitly the ultrametric distance between all-pairs of points $x$ and $y$ and that $D_{t} f(x)$ and $E_{t} f(x)$ are obtained by working on the supremum and infimum mosaics $\mathrm{Q}_{k}^{\vee} f(x)$ and $\mathrm{Q}_{k}^{\wedge} f(x)$ from the set of partitions, which is usually finite, i.e., $k=$ $0,1, \cdots, K$. 


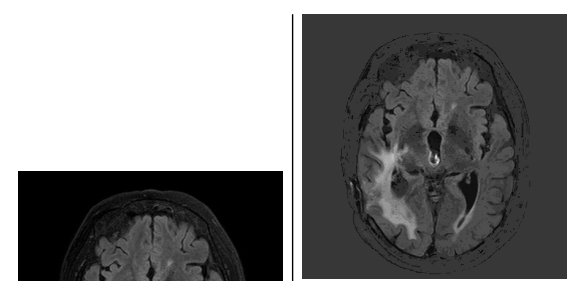

(b)

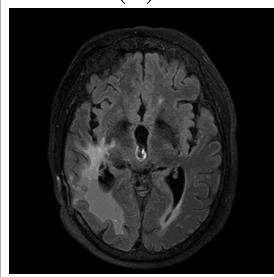

(c)

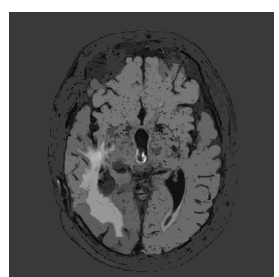

(d)

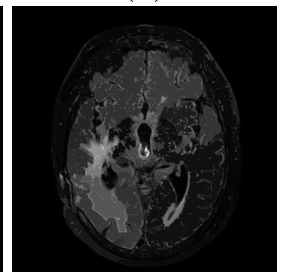

(e)

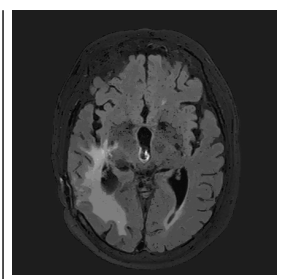

(f)

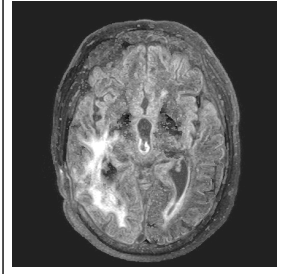

(g)

Fig. 1. Ultrametric scale-spaces: (a) original image $f(x)$, (b) and (c) ultrametric dilation $D_{t} f(x)$ with $t=0.01$ and $t=0.1,(\mathrm{~d})$ and (e) ultrametric erosion $E_{t} f(x)$ with $t=0.01$ and $t=0.1$, (f) $\infty$-mean with $t=0.01$, (g) image enhancement by $\infty$-Laplacian $f(x)-\mathcal{L}_{t}^{\infty} f(x)$ with $t=0.01$.

\subsection{Ultrametric $\infty$-mean and $\infty$-Laplacian}

Ultrametric $\infty$-mean. Given a set of $N$ points $x_{i} \in X \subset \mathbb{R}^{n}$, the $L^{\infty_{-}}$ barycenter, known as 1-center (minimax center), corresponds to the minimizer of max-of-distances function. From a geometric viewpoint, it corresponds to the center of the minimum enclosing ball of the points $x_{i}$. In the case of $\mathbb{R}$, which can be called the $\infty$-mean, the minimax center equals $m_{\infty}=\frac{1}{2}\left(\max _{1 \leq i \leq N} x_{i}+\right.$ $\left.\min _{1 \leq i \leq N} x_{i}\right)$. In our framework, the ultrametric dilation and erosion can be used to introduce the notion of ultrametric $\infty$-mean at scale $t$ just as

$$
M_{t} f(x)=\frac{1}{2}\left(D_{t} f(x)+E_{t} f(x)\right)
$$

This operator is related to the solution of the Tug-of-War stochastic game [14].

Ultrametric $\infty$-Laplacian. The infinity Laplace (or $L^{\infty}$-Laplace) operator is a 2nd-order partial differential operator. Viscosity solutions to the equation $\Delta_{\infty} u=0$ are known as infinity harmonic functions. More recently, viscosity solutions to the infinity Laplace equation have been identified with the payoff functions from randomized tug-of-war games [14]. In the case of a length spaces, there exists a conterpart, i.e.,

$$
\mathcal{L}^{\infty} u(x)=\max _{y \in \Omega, y \neq x}\left(\frac{u(y)-u(x)}{d(x, y)}\right)+\min _{y \in \Omega, y \neq x}\left(\frac{u(y)-u(x)}{d(x, y)}\right)
$$


In the case of ultrametric spaces, we introduce the multi-scale ultrametric $\infty$ Laplacian, which mimics the idea of the second-order differential operator, as follows:

$$
\begin{aligned}
\mathcal{L}_{t}^{\infty} f(x) & =\left(D_{t} f(x)-f(x)\right)-\left(f(x)-E_{t} f(x)\right) \\
& =D_{t} f(x)+E_{t} f(x)-2 f(x) .
\end{aligned}
$$

As for the standard laplacian, this operator can be used for enhancement of "edges" of function $f: f \mapsto \tilde{f}_{t}(x)=f(x)-\mathcal{L}_{t}^{\infty} f(x)$.

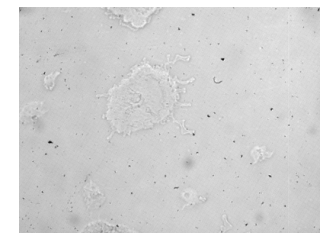

(a) $f(x)$

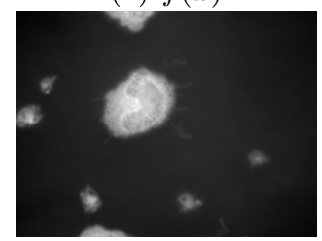

(b) for $d(x, y)$ computation

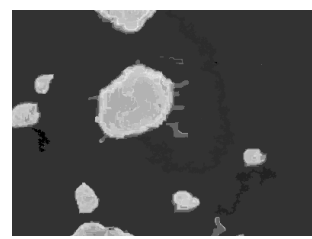

(c) $E_{t} f(x)$

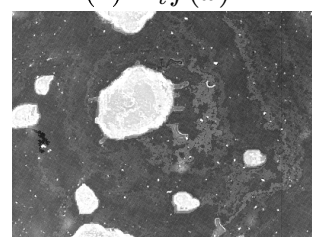

(e) $\mathcal{L}_{t}^{\infty} f(x)$

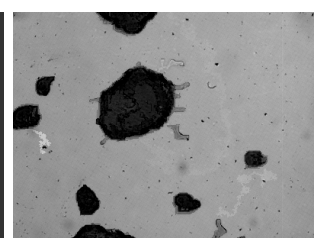

(d) $f(x)-E_{t} f(x)$

(f) $f(x)-\mathcal{L}_{t}^{\infty} f(x)$

Fig. 2. Ultrametric morphological processing of bimodal image from quantitative phase microscopy: the intensity image in (a) is processed using the ultrametric space derived from the phase image in (b), with scale parameter $t=0.005$.

\section{Applications to image and data processing}

For the examples that we consider here the ultrametric space $(X, d)$ is built from a minimum spanning tree (MST). First, let $G$ be an edge-weighted undirected neighbor graph with points $x \in X$ as vertices and all edge weights as nonnegative values. An MST of $G$ is a spanning tree that connects all the vertices together with the minimal total weighting for its edges, and let $d(x, y)$ be the largest edge weight in the path of the MST between $x$ and $y$. Then the vertices of the graph $G$, with distance measured by $d$ form an ultrametric space. By thresholding the corresponding MST at $k, 0 \leq k \leq K$, a set of partitions is obtained which produces all balls $B_{k}(x)$.

For the case of the discrete images or signals used in the examples, $G$ is 4connected pixel neighbor graph and the edge weights are the grey-level difference. In addition, a discrete ultrametric structuring function is always considered, i.e., $b_{k, t}=M-t^{-1} c_{k}$, with $c_{k}=k$. 

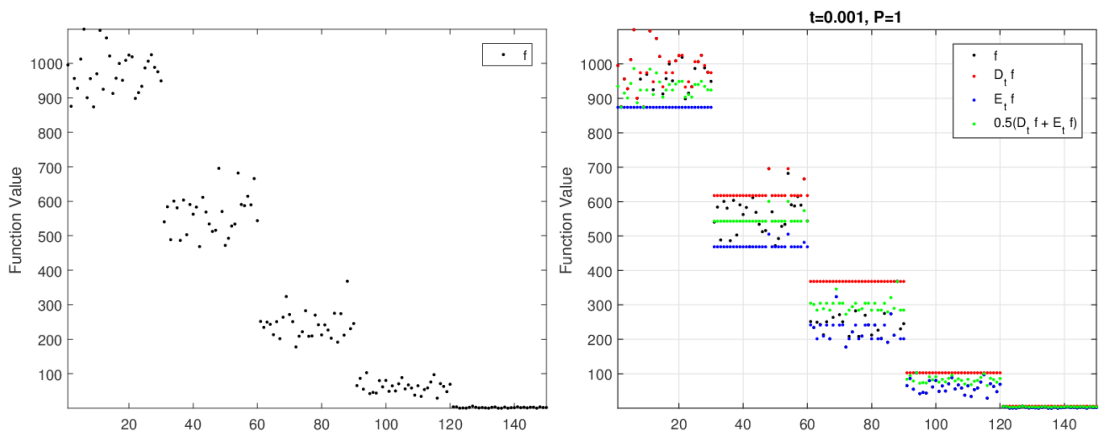

Fig. 3. Ultrametric dilation $D_{t} f(x)$ (in red), erosion $E_{t} f(x)$ (in blue) and $\infty$-mean $M_{t} f(x)$ (in green) of 1D signal $f(x)$.

The first example in Fig. 1 illustrates scale-space of ultrametric dilation $D_{t} f$ and $E_{t} f$, for two values of scale $t$. For $t=0.01$, the associated ultrametric $\infty$ mean and enhancement by $\infty$-Laplacian are also given. One can observe that the operators acting on the ultrametric balls naturally preserve the significant edges. Fig. 2 provides a case of a bimodal image from cell microscopy, where the quantitative phase image (b) is used to built the ultrametric space, and then, the intensity image (a) is ultrametrically processed using such space. A 1D signal, intrinsically organized into clusters, captured by the ultrametric point space, is used in Fig. 3. The regularization obtained by these operators can be useful in many applications of data processing.

\section{Conclusion and Perspectives}

The theory introduced in this paper provides the framework to process images or signals defined on a hierarchical representation associated to an ultrametric distance space. The effect of the operators depends on both the scale parameter and the underlying ultrametric distance. These operators have the fundamental property of acting on the function according to the pyramid of partitions associated to its ultrametric domain and therefore the notion of pixel is replaced by that of class of the partition at a given value of the hierarchy.

Ongoing work will study, on the one hand, the properties of other ultrametric structuring functions inspired from ultrametric heat kernel functions $[4,5]$ and on the other hand, the existence of a Hamilton-Jacobi PDE on ultrametric spaces using pseudo-differential operators.

\section{References}

1. J. Angulo, S. Velasco-Forero. Riemannian Mathematical Morphology. Pattern Recognition Letters, 47: 93-101, 2014. 
2. J. Angulo. Convolution in ( $\max , \min )$-algebra and its role in mathematical morphology. hal-01108121, 59 p., 2014.

3. J. Angulo. Morphological PDE and dilation/erosion semigroups on length spaces. In Proc. of ISMM'15 (12th International Symposium on Mathematical Morphology), LNCS 9082, Springer, pp. 509-521, 2015.

4. A. Bendikov, A. Grigor'yan, Ch. Pittet. On a Class of Markov Semigroups on Discrete Ultra-Metric Spaces, Potential Analysis, 37(2): 125-169, 2012.

5. A. Bendikov, A. Grigor'yan, Ch. Pittet, W. Woess. Isotropic Markov semigroups on ultrametric spaces, Uspekhi Mat. Nauk, 69(4):3-102, 2014.

6. F. Meyer. Hierarchies of Partitions and Morphological Segmentation. In ScaleSpace and Morphology in Computer Vision, Springer LNCS 2106,161-182, 2001.

7. F. Meyer, J. Stawiaski. Morphology on Graphs and Minimum Spanning Trees. In Proc. of 9th International Symposium Mathematical Morphology and Its Applications to Signal and Image Processing (ISMM'09), Springer LNCS Vol. 5720, 161-170, 2009.

8. F. Meyer. Adjunctions on the Lattice of Dendrograms. In Proc. of 9th IAPR-TC15 International Workshop on Graph-Based Representations in Pattern Recognition (GbRPR'2013), Springer LNCS Vol. 7877 , 91-100, 2013.

9. F. Meyer. Flooding Edge or Node Weighted Graphs. In Proc. of 11th International Symposium Mathematical Morphology and Its Applications to Signal and Image Processing (ISMM'13), Springer LNCS Vol. 7883, 341-352, 2013.

10. F. Meyer. Watersheds on weighted graphs, Pattern Recognition Letters, 47:72-79, 2014.

11. F. Meyer. The Waterfall Hierarchy on Weighted Graphs. In Proc. of 12th International Symposium Mathematical Morphology and Its Applications to Signal and Image Processing (ISMM'15), Springer LNCS Vol. 9082, 325-336, 2015.

12. F. Murtagh, G. Downs, P. Contreras. Hierarchical Clustering of Massive, High Dimensional Data Sets by Exploiting Ultrametric Embedding, SIAM J. Sci. Comput., 30(2):707-730, 2007.

13. L. Najman, J. Cousty, B. Perret. Playing with kruskal: Algorithms for morphological trees in edge-weighted graphs. In Proc. of 11th International Symposium Mathematical Morphology and Its Applications to Signal and Image Processing (ISMM'13), Springer LNCS Vol. 7883, 135-146 2013.

14. Y. Peres, O. Schramm, S. Sheffield, D.B. Wilson. Tug-of-war and the Infinity Laplacian. Journal of the American Mathematical Society, 22(1):167-210, 2009.

15. P. Salembier, L. Garrido. Binary Partition Tree as an Efficient Representation for Image Processing, Segmentation, and Information Retrieval, IEEE Trans. on Image Processing, 9(4):561-576, 2000.

16. J. Serra. Image Analysis and Mathematical Morphology. Vol II: Theoretical Advances, Academic Press, London, 1988. 